\title{
Characteristics of injuries associated with electric personal mobility devices: a nationwide cross-sectional study in South Korea
}

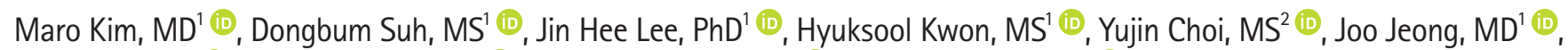 \\ Sola Kim, PhD ${ }^{3}$ (D) Soyun Hwang, MS ${ }^{4}$ (D) Joong Wan Park, MS ${ }^{4}$ (D) You Hwan Jo, PhD ${ }^{1,5}$ (i) \\ 'Department of Emergency Medicine, Seoul National University Bundang Hospital, Seongnam, Korea \\ ${ }^{2}$ Department of Emergency Medicine, Ajou University Hospital, Suwon, Korea \\ ${ }^{3}$ Department of Emergency Medicine, Hallym University Dongtan Sacred Heart Hospital, Hwaseong, Korea \\ ${ }^{4}$ Department of Emergency Medicine, Seoul National University Hospital, Seoul, Korea \\ ${ }^{5}$ Department of Emergency Medicine, Seoul National University College of Medicine, Seoul, Korea
}

Received: June 17, 2021

Revised: September 21, 2021

Accepted: October 14, 2021

\section{Correspondence to}

Dongbum Suh, MD

Department of Emergency Medicine,

Seoul National University Bundang

Hospital, 82 Gumi-ro 173beon-gil,

Bundang-gu, Seongnam 13620, Korea

Tel: +82-31-787-7594

Fax: +82-31-787-4081

E-mail:dongbums@gmail.com
Purpose: The increasing use of electric personal mobility devices (ePMDs) has been accompanied by an increasing incidence of associated accidents. This study aimed to investigate the characteristics of ePMD-related injuries and their associated factors.

Methods: This cross-sectional study was conducted using data from the Emergency Department-based Injury In-depth Surveillance database from 2014 to 2018. All patients who were injured while operating an ePMD were eligible. The primary outcome was the rate of severe injury, defined as an excess mortality ratio-adjusted Injury Severity Score of $\geq 25$. We calculated the adjusted odds ratios (AORs) of outcomes associated with ePMD-related injuries.

Results: Of 1,391,980 injured patients, 684 (0.05\%) were eligible for inclusion in this study. Their median age was 28 years old, and most injuries were sustained by men (68.0\%). The rate of ePMD-related injuries increased from 3.1 injuries per 100,000 population in 2014 to 100.3 per 100,000 population in 2018. A majority of the injuries occurred on the street (32.7\%). The most commonly injured area was the head and face (49.6\%), and the most common diagnosis was superficial injuries or contusions (32.9\%). Being aged 55 years or older (AOR, 3.88; 95\% confidence interval, 1.33-11.36) and operating an ePMD while intoxicated (AOR, 2.78; 95\% confidence interval, 1.52-5.08) were associated with severe injuries.

Conclusions: The number of emergency room visits due to ePMD-related injuries is increasing. Old age and drunk driving are both associated with serious injuries. Active traffic enforcement and safety regulations regarding ePMDs should be implemented to prevent severe injuries caused by ePMD-related accidents.

Keywords: Injury Severity Score; Accidents; Traumatic brain injuries; Epidemiology; Wounds and injuries 


\section{INTRODUCTION}

Electric personal mobility devices (ePMDs) are electrically powered wheeled devices that provide personal transport. These devices provide simple, fast, and convenient transportation and are attracting considerable interest as a novel tool for short-haul transport and leisure activities [1].

The demand for ePMDs has increased rapidly in South Korea in recent years, and their increasing use has been accompanied by an increasing incidence of ePMD-related injuries [2-6]. However, the epidemiologic characteristics of ePMD-related injuries have only been studied in a few countries. In one study from Singapore, ePMD-related injuries were generally found not to be severe and were primarily external wounds and upper and lower limb injuries [7]. In a Swedish study, most of the reported ePMD-related injuries resulted from the driver hitting the ground due to device turnover [8]. Few studies on ePMD-related injuries have been conducted in South Korea, however.

Under South Korea's Road Traffic Act, ePMDs are classified as "motorized bicycles", and their operation is prohibited outside of roadways [9]. Furthermore, a driver's license and helmet are required for their use. However, many ePMD owners operate them outside of roadways to avoid collisions with motor vehicles and rarely wear helmets during use $[10,11]$. These behaviors can lead to severe injuries. This study aimed to examine the characteristics of ePMD-related injuries and identify risk factors associated with severe ePMD-related injuries in South Korea.

\section{METHODS}

The present study protocol was reviewed and approved by the Institutional Review Board of the Seoul National University Bundang Hospital (No. X-1903-528-902).

\section{Study design}

This cross-sectional study was performed using data from the Emergency Department-based Injury In-depth Surveillance (EDIIS) database sponsored by the Korea Disease Control and Prevention Agency (KDCA). The EDIIS is a prospective nationwide injury database that stores data on patients with injuries who present to the emergency rooms (ERs) of specific representative medical institutions in Korea.

\section{Data source}

In total, 17 (in 2014), 20 (in 2015), and 23 (from 2016 to 2018) tertiary academic hospitals shared data on patients with injuries who presented to ERs using the EDIIS database. The EDIIS database includes demographic, injury prevention- related, and epidemiologic information as well as pre-hospital procedures, initial clinical findings from the ER visit, diagnoses (coded using the International Classification of Diseases, 10th Revision [ICD-10]), treatments, dispositions, and patient outcomes after admission. Individual incidents were described in written Korean. Primary information was obtained by physicians from each medical institution during clinical practice and by trained coordinators from the EDIIS project assigned to each hospital. The coordinators collected the data from a standardized registry. The data from each ER were registered on a web-based database hosted by the KDCA, and a quality improvement program was conducted regularly [12].

\section{Selection of participants}

Among all injuries reported in the EDIIS database between January 2014 and December 2018, only patients who were injured while operating an ePMD were included in this study. To identify ePMD-related injuries, we searched the database with ePMD-related keywords, including "electric scooter", "e-scooter", "electric kickboard", "e-kickboard", "hoverboard(s)", "electric unicycle", "e-unicycle", "Segway", "Lime scooter", and "Ninebot", and two researchers reviewed the incident descriptions. After reviewing each entry, data were excluded if 1 ) the injury was caused by an ePMD used as an aid for a disabled person, such as an electric wheelchair or mobility scooter, or 2) the accident was not related to the operation of the ePMD.

\section{Variables and measurements}

This study collected information for each injury on age, sex, injury date, device type, injury mechanism, injury location, the rider's state of intoxication, helmet use, diagnosis (ICD-10 code), and disposition. Age was classified as 0 to 14,15 to 24,25 to 34 , 35 to 44,45 to 54 , and 55 years or older, based on previous studies [13]. An "e-scooter" was defined as a device powered by an electric motor with wheels and handlebars that is designed to be stood upon by the operator. An "electric unicycle" and "hoverboard" were defined as narrow, horizontal boards with one or two wheels, respectively, that move when the rider leans forward. Mechanisms of injury were categorized as falloff, collision with a motor vehicle, collision with a human, collision with another ePMD, or other. Possible injury locations were indoors, public property (a car-free public facility), street (a thoroughfare for cars), sidewalk (a foot traffic-only pathway), bike-way (a bicycle-only pathway), driveway/ parking lot, and alley (a road 
without sidewalks). Anatomical injury sites were categorized as head and face, neck, torso (including the thorax, abdomen, back, pelvis, and genitals), and upper and lower extremities (including the shoulders, upper arms, elbows, forearms, wrists, hips, thighs, knees, lower legs, ankles, and feet) according to the ICD10 codes for the injury mortality diagnosis matrix by the Centers for Disease Control and Prevention of the United States [14].

To assess injury severity, the excess mortality ratio-adjusted Injury Severity Score (EMR-ISS) was calculated using ICD-10 codes. The EMR-ISS classifications used in this study were mild (scores 1-8), moderate (scores 9-24), severe (scores 25-74), or critical (scores $\geq 75$ or death), as in a previous study [15].

\section{Study outcomes}

The primary outcome of the study was the incidence of severe injury. The secondary outcome was the incidence of acute traumatic brain injury, defined by ICD-10 codes of S02.0xx, S02.1, S06.2, and S06.3x [16]. The tertiary outcome was the rate of intensive care unit (ICU) admission.

\section{Statistical analysis}

All statistical analyses were performed using STATA version 14.2 (StataCorp LP, College Station, TX, USA). Continuous variables were presented as medians with interquartile ranges (IQR), and categorical variables were presented as frequencies with percentages. To identify statistically significant differences between the outcome groups, we used the Wilcoxon rank-sum test for continuous variables and the chi-square test or Fisher exact test for categorical variables. Odds ratios (ORs) with 95\% confidence intervals (CI) were calculated using multivariate logistic regression analysis to evaluate the factors associated with the outcomes. The level of statistical significance was defined as a P-value of $\leq 0.05$.

\section{RESULTS}

Incidence and characteristics of ePMD-related injuries Of the 1,391,980 patients injured between 2014 and 2018, 1,472 patients $(0.11 \%)$ had records that included ePMD-related keywords in their descriptions. After excluding ineligible cases, 684 cases $(0.05 \%)$ were ultimately used in the final analysis. Among them, 505 cases $(73.8 \%)$ were related to e-scooters and 179 cases (26.2\%) were related to electric hoverboards (Fig. 1).

Fig. 2 shows the trends in ePMD-related injuries among the study population. The rate of ePMD-related injuries due to e-scooters or hoverboards increased from 3.1 ePMD-related ER visits per 100,000 population in 2014 to 100.3 per 100,000 population in 2018.

Table 1 shows demographic characteristics and clinical features according to ePMD type. The median age was 28 years old (IQR, 19-38 years old) and a higher proportion of men (68.0\%) sustained injuries related to ePMDs. Patients who were injured while operating electric hoverboards tended to be younger than those who were injured while operating e-scooters. The distribution of the time of injury was different between the two groups. The rate of helmet use was low in both groups, at 3.4\% for the e-scooter group and 2.2\% for the hoverboard group $(\mathrm{P}=0.210)$, and operation while intoxicated was more common in the e-scooter group than in the hoverboard group. Fall-off injuries were the most common injury mechanism, at $69.9 \%$ in the e-scooter group and $83.8 \%$ in the hoverboard group; however, collisions with vehicles or stationary objects were more fre-

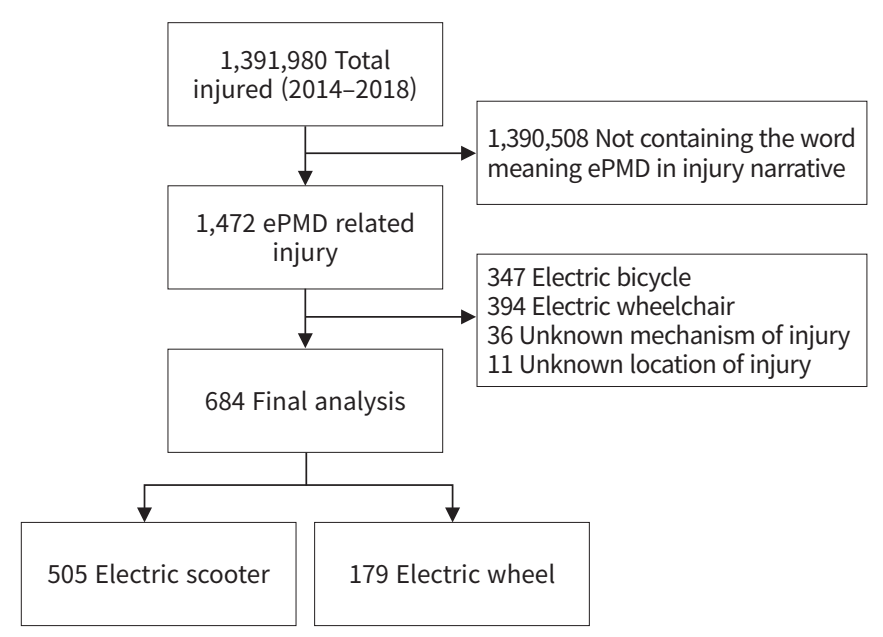

Fig. 1. Study population. ePMD, electric personal mobility devices.

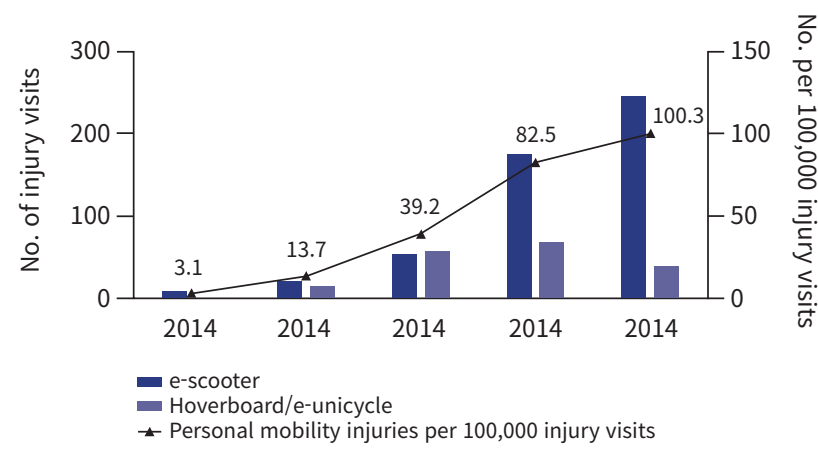

\begin{tabular}{lrrrrr|}
\hline Total injury visits & 259,752 & 270,384 & 283,422 & 294,408 & 284,014 \\
\hline Participating hospitals & 17 & 20 & 23 & 23 & 23 \\
\hline
\end{tabular}

Fig. 2. Annual number of injuries related to electric personal mobility devices. 
Table 1. Demographic findings and clinical outcomes of the study population by ePMD type

\begin{tabular}{|c|c|c|c|c|}
\hline Characteristics & Total $(\mathrm{n}=684)$ & E-scooter $(n=505)$ & Hoverboard/e-unicycle $(\mathrm{n}=179)$ & $\mathrm{P}$-value \\
\hline Age (yr) & & & & $<0.001$ \\
\hline $0-14$ & $133(19.4)$ & $73(14.5)$ & $60(33.5)$ & \\
\hline $25-34$ & $157(23.0)$ & $125(24.8)$ & $32(17.9)$ & \\
\hline $35-44$ & $151(22.1)$ & $115(22.8)$ & $36(20.1)$ & \\
\hline $45-54$ & $57(8.3)$ & $44(8.7)$ & $13(7.3)$ & \\
\hline Median (interquartile range) & - & $29(21-38)$ & $26(12-39)$ & 0.026 \\
\hline Male sex & $465(68.0)$ & $350(69.3)$ & $115(64.2)$ & 0.210 \\
\hline Year of injury & & & & $<0.001$ \\
\hline 2014 & $8(1.2)$ & $8(1.6)$ & 0 & \\
\hline 2015 & $37(5.4)$ & $21(4.2)$ & $16(8.9)$ & \\
\hline Day of injury (weekend) & $298(43.6)$ & $216(42.8)$ & $82(45.8)$ & 0.480 \\
\hline Time of injury & & & & 0.012 \\
\hline $0-6 \mathrm{AM}$ & $180(26.3)$ & $147(29.1)$ & $33(18.4)$ & \\
\hline 6-12 AM & $35(5.1)$ & $29(5.7)$ & $6(3.4)$ & \\
\hline $12-6 \mathrm{PM}$ & $204(29.8)$ & $146(28.9)$ & $58(32.4)$ & \\
\hline 6-12 PM & $265(38.7)$ & $183(36.2)$ & $82(45.8)$ & \\
\hline Helmet use & $21(3.1)$ & $17(3.4)$ & $4(2.2)$ & 0.210 \\
\hline Drunk driving (alcohol) & $70(10.2)$ & $64(12.7)$ & $6(3.4)$ & $<0.001$ \\
\hline EMS use & $267(39.0)$ & $226(44.8)$ & $41(22.9)$ & $<0.001$ \\
\hline Mechanism of injury & & & & 0.002 \\
\hline Location of injury & & & & $<0.001$ \\
\hline Indoor & $41(6.0)$ & $21(4.2)$ & $20(11.2)$ & \\
\hline Public property & $109(15.9)$ & $65(12.9)$ & $44(24.6)$ & \\
\hline Street & $224(32.7)$ & $180(35.6)$ & $44(24.6)$ & \\
\hline Sidewalk & $137(20.0)$ & $109(21.6)$ & $28(15.6)$ & \\
\hline Bike-way & $29(4.2)$ & $22(4.4)$ & $7(3.9)$ & \\
\hline Driveway/parking & $8(1.2)$ & $6(1.2)$ & $2(1.1)$ & \\
\hline Alley & $136(19.9)$ & $102(20.2)$ & $34(19.0)$ & \\
\hline Injury severity, EMR-ISS & & & & $<0.001$ \\
\hline Mild & $233(34.1)$ & $150(29.7)$ & $83(46.4)$ & \\
\hline Moderate & $363(53.1)$ & $276(54.7)$ & 87 (48.6) & \\
\hline Severe & $82(12.0)$ & $74(14.7)$ & $8(4.5)$ & \\
\hline Critical & $6(0.9)$ & $5(1.0)$ & $1(0.6)$ & \\
\hline
\end{tabular}

(Continued to the next page) 
Table 1. Continued

\begin{tabular}{|c|c|c|c|c|}
\hline Characteristics & Total $(\mathrm{n}=684)$ & E-scooter $(n=505)$ & Hoverboard/e-unicycle $(\mathrm{n}=179)$ & P-value \\
\hline Operation (yes) & $60(8.8)$ & $47(9.3)$ & $13(7.3)$ & 0.41 \\
\hline 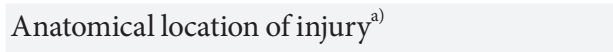 & & & & $<0.001$ \\
\hline Head and face & $332(49.6)$ & $271(55.0)$ & $61(34.5)$ & \\
\hline Neck & $11(1.6)$ & $7(1.4)$ & $4(2.3)$ & \\
\hline Torso & $30(4.5)$ & $22(4.5)$ & $8(4.5)$ & \\
\hline Extremities & $297(44.3)$ & $193(39.1)$ & $104(58.8)$ & \\
\hline Diagnosis $^{\text {a) }}$ & & & & 0.326 \\
\hline Fracture & $264(28.6)$ & $200(27.4)$ & $64(33.3)$ & \\
\hline Dislocation & $38(4.1)$ & $30(4.1)$ & $8(4.2)$ & \\
\hline Internal organ injuries & $80(8.7)$ & $61(8.4)$ & $19(9.9)$ & \\
\hline Open wound & $232(25.2)$ & $193(26.4)$ & $39(20.3)$ & \\
\hline Superficial injury or contusion & $303(32.9)$ & $242(33.2)$ & $61(31.8)$ & \\
\hline Amputation, blood vessel injuries, or crushing & $5(0.5)$ & $4(0.6)$ & $1(0.5)$ & \\
\hline Disposition & & & & 0.075 \\
\hline Discharge & $584(85.4)$ & $422(83.6)$ & $162(90.5)$ & \\
\hline Transfer & $15(2.2)$ & $12(2.4)$ & $3(1.7)$ & \\
\hline Admission & $85(12.4)$ & $71(14.1)$ & $14(7.8)$ & \\
\hline
\end{tabular}

Values are presented as number (\%).

EMS, emergency medical services; ePMD, electric personal mobility devices; EMR-ISS, excess mortality ratio-adjusted Injury Severity Score.

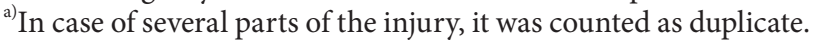

quent in the e-scooter group than in the hoverboard group. A higher proportion of injuries in the e-scooter group had EMRISS classifications of moderate or severe, and the e-scooter group also had a higher ICU admission rate than the hoverboard group. One death was recorded in the e-scooter group.

Table 2 shows the clinical results and demographic findings by age group. Injuries related to e-scooters were more common than injuries related to hoverboards across all age groups. The rate of emergency medical service utilization was highest among those aged $>55$ years old. Those aged 0 to 14 years old experienced the most injuries on public property, while the majority of injuries occurred on the street or sidewalk for the other age groups.

\section{Main outcomes}

The results of the multivariate logistic regression models are shown in Table 3. The occurrence of severe injury, traumatic brain injury, and ICU admission was higher among those aged 55 and older. There were no cases of traumatic brain injury or ICU admission when the rider wore a helmet. The likelihood of a severe injury (i.e., with an EMR-ISS of $\geq 25$ ) was higher for men and for patients who had consumed alcohol before riding (adjusted OR [AOR], 2.11; 95\% CI, 1.14-3.90 for men and AOR, 2.78; 95\% CI, 1.52-5.08 for those with alcohol consumption).
Moreover, patients who sustained injuries on roads or streets (AOR, 2.68; 95\% CI, 1.11-6.45) were more likely to be admitted to the ICU than patients injured elsewhere.

\section{DISCUSSION}

Thus far in South Korea, only one study on ePMD-related injuries has been conducted, and it only examined injuries at one center. The present study analyzed multicenter data collected from evenly distributed, representative medical institutions in South Korea to examine the demographics of patients injured by ePMDs [17].

As ePMD use has grown in popularity worldwide, the burden of ePMD-related injuries has similarly increased. In Singapore, the incidence of ePMD-related injuries increased by $68 \%$ over 3 years [7]. Similarly, we found that the rate of ePMD-related injuries in South Korea also increased rapidly, from 3.1 injuries per 100,000 population in 2014 to 100.3 injuries per 100,000 population in 2018. Despite this increase in ePMD-related injuries, there have been few changes in legislation and infrastructure in South Korea. The Korean Road Traffic Act has not yet been updated to distinguish this new means of personal transport from motorcycles, which has led to an increase in the number of citizens who defy traffic laws. This can lead to an increase in traffic 
Table 2. Demographic findings and clinical outcomes of the study population by age group

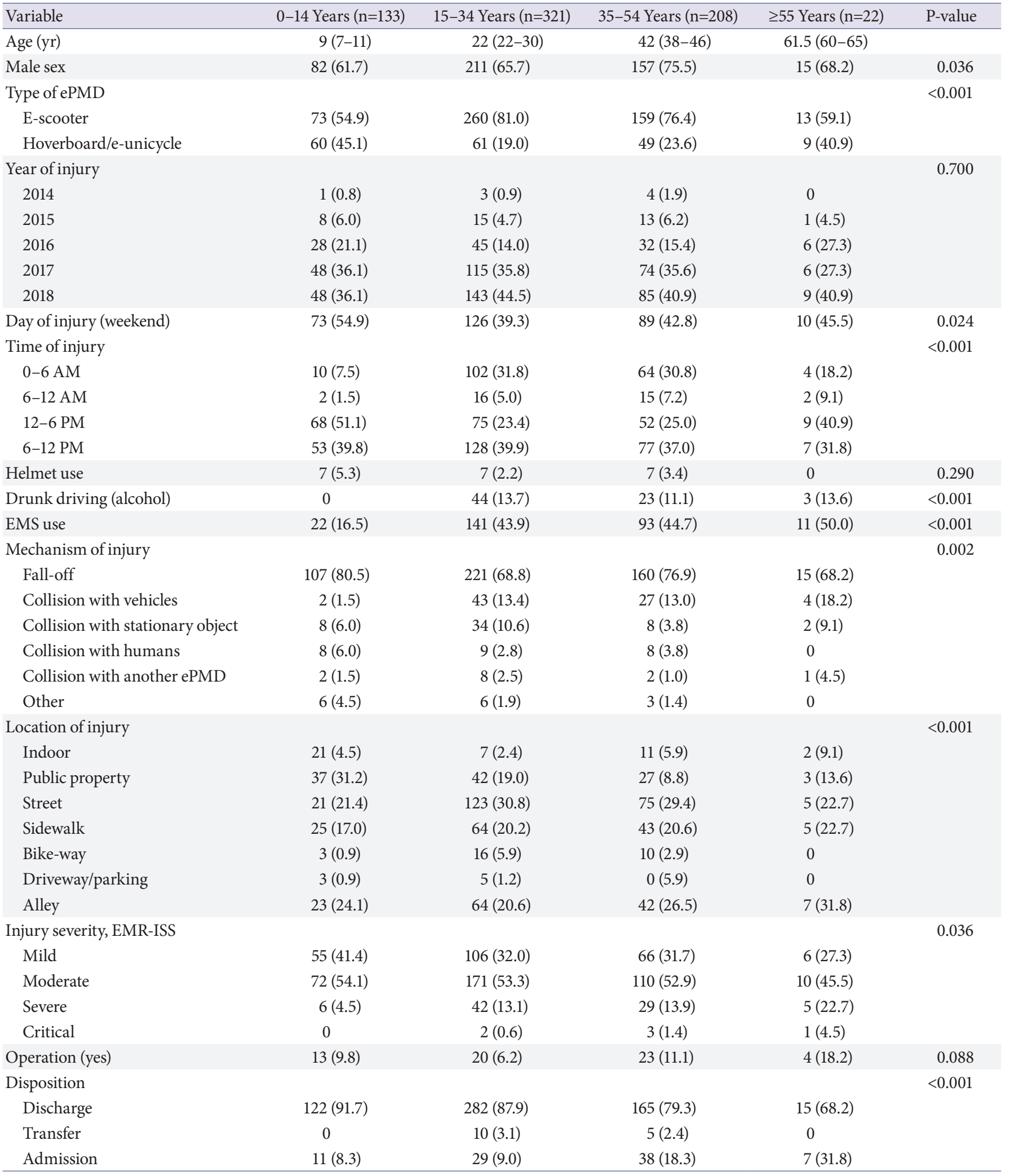

Values are presented as median (interquartile range) or number (\%).

ePMD, electric personal mobility devices; EMS, emergency medical services; EMR-ISS, the excess mortality ratio-adjusted Injury Severity Score. 




accidents and injuries resulting from the operation of ePMDs. Therefore, to prevent accidents and minimize injuries, the characteristics of ePMD-related injuries must be analyzed in order to create new regulations and infrastructure to address this issue.

We classified the most popular types of ePMDs into e-scooters and hoverboards or e-unicycles. Injuries related to e-scooters were more common than injuries related to hoverboards or e-unicycles in 2017 and 2018. Furthermore, injuries related to e-scooters were more likely to involve motor vehicle collisions than injuries related to hoverboards or e-unicycles, and they were also more likely to be severe and lead to hospital admission.

Wearing a helmet is one of the most important safety precautions required by law when operating an ePMD. One of the most remarkable findings in this study was the low rate of helmet use across all age groups. In this study, there were no cases of traumatic brain injury or admission to the ICU when the patient had been wearing a helmet. Given this finding, it is essential for riders to wear a helmet when operating ePMDs.

Injuries under the influence of alcohol accounted for approximately $10 \%$ of all cases, and intoxication was significantly related to severe injury. At present, operating an ePMD while intoxicated is illegal, and greater measures must be taken to enforce this law. The incidence of ePMD-related injuries on roads and streets was associated with admission to the ICU. Currently, the law in Korea requires ePMDs to be operated only on roadways. Considering that operation on sidewalks can also lead to accidents, particularly involving pedestrians, laws regarding the operation of ePMDs must be reevaluated for optimal safety. If necessary, new infrastructure for ePMDs should be considered in addition to roadways.

One limitation of this study is that the number of ePMD-related injuries was small (684 cases). However, as this study used data from representative regional medical institutions, most severe injuries that required specialized treatment were likely included in the EDIIS database. Therefore, we believe that the data are representative of the population of South Korea. Another limitation is that data related to fatalities may have been missing since, if the rider had already died, he or she would not have been transferred to one of the medical institutions from this study. Therefore, further studies on ePMD-related injuries based on pre-hospitalization data should be conducted to examine incidents in which riders are not hospitalized.

In conclusion, there has been a recent increase in ER visits in South Korea due to ePMD-related injuries. Wearing a helmet 
while operating an ePMD is essential, as doing so can reduce the risk of traumatic head injury and ICU treatment. Drunk driving increases the likelihood of serious injury, and active administrative enforcement targeting the illegal operation of ePMDs should be increased. Furthermore, regulations related to the operation of ePMDs should be updated to prevent serious injuries from ePMD-related accidents. The results of this study may help inform the development of policies to prevent ePMD-related injuries.

\section{NOTES}

\section{Ethical statement}

The present study protocol was reviewed and approved by the Institutional Review Board of the Seoul National University Bundang Hospital (No. X-1903-528-902). The requirement for written informed consent was waived by the Institutional Review Board.

\section{Conflicts of interest}

The authors have no conflicts of interest to declare.

\section{Funding}

None.

\section{Author contributions}

Conceptualization: MK, DS, JWP, YHK; Study design: MK, DS, JWP, YHK; Acquisition, analysis, or interpretation of data: MK, DS, JJ, SK; Statistical analysis: DS; Writing-original draft: MK, DS; Writing-review\&editing: JHL, HK, YC, JWP, YHJ. All authors read and approved the final copy of the manuscript.

\section{REFERENCES}

1. Choron RL, Sakran JV. The integration of electric scooters: useful technology or public health problem. Am J Public Health 2019;109:555-6.

2. Shin GW, Lee KJ, Park D, Lee JH, Yun MH. Personal mobility device and user experience: a state-of-the-art literature review. Proceedings of the Human Factors and Ergonomics Society Annual Meeting 2018;62:1336-7.

3. Shin H, Lee J, Kim S. Study on the improvement of laws and regulations for personal mobilities [Internet]. Sejong: The Korea Transport Institute 2016 [cited 2021 Mar 29]. Available from: https://www.koti.re.kr/component/file/ND_fileDownload.do?q_fileSn = 103385\&q_fileId $=7 f 1450 a 3-40 d 6-4 a 7 f-$ 8da2-65fa12e874c1.

4. Boniface K, McKay MP, Lucas R, Shaffer A, Sikka N. Serious injuries related to the Segway ${ }^{\circledR}$ personal transporter: a case series. Ann Emerg Med 2011;57:370-4.

5. Ashurst J, Wagner B. Injuries following segway personal transporter accidents: case report and review of the literature. West J Emerg Med 2015;16:693-5.

6. Roider D, Busch C, Spitaler R, Hertz H. Segway ${ }^{\oplus}$ related injuries in Vienna: report from the Lorenz Böhler Trauma Centre. Eur J Trauma Emerg Surg 2016;42:203-5.

7. Tan AL, Trauma Coordinators and Trauma Service Representatives; Nadkarni N, Wong TH. The price of personal mobility: burden of injury and mortality from personal mobility devices in Singapore: a nationwide cohort study. BMC Public Health 2019;19:880.

8. Carlsson A, Lundalv J. Acute injuries resulting from accidents involving powered mobility devices (PMDs): development and outcomes of PMD-related accidents in Sweden. Traffic Inj Prev 2019;20:484-91.

9. Korean Law Information Center. Road traffic Act 2018 [Internet]. Sejong: Korean Law Information Center 2018 [cited 2019 Feb 3]. Available from: http://www.law.go.kr/LSW/eng/ engLsSc.do? menuId = 2\&query $=\#$ liBgcolor 14 .

10. Trivedi TK, Liu C, Antonio AL, et al. Injuries associated with standing electric scooter use. JAMA Netw Open 2019;2: e187381.

11. Robinson T, Agarwal M, Chaudhary S, Costello BE, Simon HK. Pediatric hoverboard injuries: a need for enhanced safety measures and public awareness. Clin Pediatr (Phila) 2016; 55:1078-80.

12. Park GJ, Ro YS, Shin SD, Song KJ, Hong KJ, Jeong J. Preventive effects of car safety seat use on clinical outcomes in infants and young children with road traffic injuries: a 7-year observational study. Injury 2018;49:1097-103.

13. Skaansar O, Tverdal C, Ronning PA, et al. Traumatic brain injury: the effects of patient age on treatment intensity and mortality. BMC Neurol 2020;20:376.

14. Centers for Disease Control and Prevention. CORRECTED Table II. ICD-10 codes used to define cells of the injury mortality diagnosis (IMD) matrix for use with multiple-cause-ofdeath data [Internet]. Atlanta (GA): Centers for Disease Control and Prevention 2006 [cited 2021 Sep 1]. Available from: https://www.cdc.gov/nchs/data/ice/10_diamatrix.pdf.

15. Kim J, Shin SD, Im TH, et al. Development and validation of the Excess Mortality Ratio-adjusted Injury Severity Score using the International Classification of Diseases 10th edition. 
Acad Emerg Med 2009;16:454-64.

16. Khajanchi MU, Kumar V, Warnberg Gerdin L, et al. Prevalence of a definitive airway in patients with severe traumatic brain injury received at four urban public university hospitals in India: a cohort study. Inj Prev 2019;25:428-32.

17. Kim YW, Park WB, Cho JS, Hyun SY, Lee G. The new recreational transportation on the street: personal mobility, is it safe? J Trauma Inj 2018;31:125-34. 